\title{
Sonography Cervical Assessment in Multiple Pregnancy in Correlation with Gestational Age
}

\author{
Mahrous Areej ${ }^{1}$, Wesam Kurdi², Samar Nahas ${ }^{3}$ \\ ${ }^{1}$ Department of Obstetrician and Gynecologists, King Salman Hospital, Riyadh, Saudi Arabia \\ ${ }^{2}$ Department of Obstetrician and Gynecologists, King Faisal Specialist Hospital and Research Centre, Riyadh, Saudi Arabia \\ ${ }^{3}$ Department of Obstetrician and Gynecologists, University of California, Riverside, California, USA \\ Email: Mahrous_a@yahoo.com,kurdi@kfshrc.edu.sa, Nahasmd@yahoo.ca
}

How to cite this paper: Areej, M., Kurdi, W. and Nahas, S. (2022) Sonography Cervical Assessment in Multiple Pregnancy in Correlation with Gestational Age. Open Journal of Obstetrics and Gynecology, 12, 113-117. https://doi.org/10.4236/ojog.2022.122011

Received: January 2, 2022

Accepted: February 5, 2022

Published: February 8, 2022

Copyright $\odot 2022$ by author(s) and Scientific Research Publishing Inc. This work is licensed under the Creative Commons Attribution International License (CC BY 4.0).

http://creativecommons.org/licenses/by/4.0/

\begin{abstract}
Objective: Observe and correlate the cervical length by ultrasound and likelihood of cervical incompetence and premature delivery in multiple gestations. Materials and Methods: Retrospective study in multiple pregnancy (twin, triplet, quadruplet) delivered between 2002-2003, in King Faisal Specialist Hospital and Research Centre, Riyadh, Saudi Arabia. A total of 68 multiple pregnancies underwent routine ultrasound for fetal assessment between 16 - 32 weeks when the cervix was observed. The sonographic measurements included cervix $>2.5 \mathrm{~cm}$ and $<2.5 \mathrm{~cm}$. The time of ultrasound was divided in five groups by gestational age from 18 - 20 weeks, $21-24$ weeks, $25-26$ weeks, $29-32$ weeks and $>32$ weeks. The gestational age at delivery was our outcome parameter. The data was analyzed statistically using Fisher's exact test and P-value below 0.05 was considered significant. Results: Endovaginal ultrasongoraphic cervical measurement in multiple pregnancies predicted increased preterm delivery risk regardless of maternal age, previous history of preterm deliveries or presence of vaginal infection. It was found that spontaneous preterm labor $<32$ weeks is a relatively rate outcome with cervix $>25$ $\mathrm{mm}$; this majority reached $>32$ weeks while the patient who had cervix $<25$ $\mathrm{mm}, 1$ in 25 of the women who underwent a cervical ultrasound assessment will have preterm labor. The finding in ultrasound justifies the results of higher aggressive management inpatient with cervix $<25 \mathrm{~mm}$. Conclusion: Cervical assessment during routine ultrasound in multiple gestations seems to be useful for prediction of preterm delivery and counseling the patient without risk factors regarding cervical cerclage. Because of restricted number of patients, we suggest further study with bigger sample and prospective trial for valued conclusions.
\end{abstract}

\section{Keywords}

Sonography, Multiple Pregnancy, Cervical Assessment 


\section{Introduction}

Risk assessment for preterm delivery remains difficult, particularly among women with no prior history of preterm birth. We hypothesized that accurate assessment of cervical length by endovaginal ultrasonography could predict preterm delivery risk in multiple pregnancy. Recent advances in infertility treatments have created many more multiple gestations than in the past when it was rate without the aid of infertility treatment. Twins occur once in 83 deliveries; triplets occur in every 6900 deliveries and quadruplet occur in every 575,000 deliveries but fertility techniques have certainly increased rates [1]. The most serious complication of multiple gestations is preterm delivery. Each baby is added to the pregnancy that takes approximately three and a half weeks from the average time of delivery. Babies can survive as early as $23-24$ weeks, but not all babies survive and not survivors are healthy [1]. Ultrasound is a critical tool in management of multiple gestation. Special attention needs to be paid to the cervix. This vaginal ultrasound is important; because current shortening is silent, you may not feel any change until it is too late [1]. The best time for delivery is as closed as to 38 weeks in twin; with triplet, 35 to 36 weeks and with quadruplet, 34 to 35 weeks [1]. The American College of Obstetricians and Gynecologists (ACOG) recently published a clinical management guideline on cervical insufficiency; serial ultrasound assessments of the cervix in low-risk women have demonstrated low sensitivity and low positive predictive values, meaning ultrasonography lacks enough discriminatory power to recommend routine use [2]. Several study results suggest that transvaginal ultrasound considered in multiple pregnancies, because the upper portion of the cervix is not easily distinguished from the lower uterine segment in early pregnancy. These assessments should not begin before 16 to 20 weeks of gestation. Preterm birth complicates from $8 \%$ - $10 \%$ of all births; most deaths of premature infants occur in infants before the 32 weeks of pregnancy [3]. The researchers found that women whose cervices had shortened to less than $25 \mathrm{~mm}$ in length by the time of the first ultrasound evaluation were 3.3 times more likely to give birth prematurely than the other women in the study [3]. The aim of the present study was to describe our experience for cervical length assessment by endovaginal sonography, predict spontaneous preterm birth before 32 weeks gestation in multiple pregnancy.

\section{Methods}

Retrospective Study - Between 2002-2003 for 68 patients with multiple gestation (Tw in ${ }^{58}$, Triplet7, Quadruplet ${ }^{1}$ ) followed up at King Faisal Specialist Hospital \& Research Centre underwent serial cervical ultrasound assessment from $16-20$ weeks, 21 - 24 weeks, 25 - 28 weeks, 29 - 32 weeks and $>32$ weeks gestation where cervix observed as $>25 \mathrm{~mm}$ and less $<25 \mathrm{~mm}$. Data collected included information about age, obstetric history, presence of vaginal infection, previous history of second trimester abortion, or cervical suture. Demographic and antenatal data were collected retrospectively from the labor and delivery log book and patient files. 
Transvaginal ultrasonographic assessment of the cervix was performed. The ultrasound images were analyzed to assess changes in the cervix, are associate with spontaneous premature delivery in multiple gestation $<32$ weeks as primary outcome by the time of first ultrasound evaluation. Statistics used where Fisher's Exact Test. Significance was considered at the .05 level. Exclusion criteria for single twin pregnancy or any multiple pregnancy who did not had cervical assessment during pregnancy or had late booking $>32$ weeks or last follow-up and delivered outside the hospital. Also, induction of labor $<32$ weeks due to intrauterine death or preeclampsia excluded.

\section{Results}

Among 86 patients were enrolled, 18 patients excluded from the analysis because either they did not undergo cervical sonography during pregnancy or they lost follow-up or they delivered outside. 68 patients whose cervices were measured ultrasonographically at 16 - 20 weeks, 21 - 24 weeks, 25 - 28 weeks, 29 - 32 weeks and $>32$ weeks. The mean gestational age where the cervices were measured (20.04). The duration of pregnancy according to whether the cervical length was $<25 \mathrm{~mm}$ or $>25 \mathrm{~mm}$. It was found that spontaneous preterm birth of multiple pregnancy $<32$ weeks is a relatively rare outcome with long cervix $>25 \mathrm{~mm}$. The rate of spontaneous delivery between $18-20$ weeks was $0 \%$ among the women who had vaginal sonographic assessment for cervix $>2.5 \mathrm{~cm}$. $4.41 \%$ who had delivery between 21 - 24 weeks, $1.4 \%$ who had delivery between 25 - 28 weeks and $7.35 \%$ who had delivery between 29 - 32 weeks. The majority reached $>32$ weeks among the patient who had cervix $>25 \mathrm{~mm}$ while the patient who had cervix < $25 \mathrm{~mm} .8 .8 \%$ who had achieved delivery $>32$ weeks with significant PS 0.0053 (Table 1). Endovaginal ultrasonographic cervical measurement in multiple pregnancies predicted increased preterm delivery risk regardless of maternal age, previous history of preterm deliveries presence of vaginal infection or previous cervical suture.

\section{Discussion}

Due to high risk of preterm multiple gestational delivery with short cervix $<2.5$ $\mathrm{cm}$ ultrasound finding justify the result of higher aggressive management inpatient with high-risk group including cervical cerclage. Our findings confirmed those of previous studies that have found as inverse relation between the length of cervix as measured by ultrasonography during pregnancy and the pregnancy of preterm delivery. General incompetence is difficult to ascertain because the clinical history and physical finding are variable and subjective. Many women with prior pregnancy losses or early preterm labor present no clear history of cervical incompetence.

However, sonogram can provide an objective method to assess the anatomy of the cervix and the internal os [4]. Kushnir et al. assessed current length using vaginal ultrasonogarphy and applied the normative cervical length to active antepartum management for early detection and prevention of preterm delivery [4]. 
Table 1. Gestation age at delivery.

\begin{tabular}{ccccccc}
\hline Frequency Percent & $\mathbf{1 6 - 2 0}$ & $\mathbf{2 1 - 2 4}$ & $\mathbf{2 5 - 2 8}$ & $\mathbf{2 9 - 3 2}$ & $\mathbf{3 2}$ & Total \\
\hline $\mathbf{2} 2.5$ & 0 & 3 & 1 & 5 & 50 & 59 \\
& 0.00 & 4.41 & 1.47 & 7.35 & 73.53 & 86.75 \\
$<2.5$ & 1 & 1 & 1 & 0 & 6 & 9 \\
& 1.47 & 1.47 & 1.47 & 0.00 & 8.82 & 13.24 \\
Total & 1 & 4 & 2 & 6 & 56 & 68 \\
& 1.47 & 5.88 & 2.94 & 7.36 & 82.35 & 100.00 \\
\hline
\end{tabular}

Fisher's Exact Test $=$ P -0.0053. The FREQ Procedure Table of Find by GA Delivery.

To avoid empirical use of overuse of cervical cerclage, a new method has been described using vaginal ultrasound and trans fundal pressure to evaluate the asymptomatic incompetent cervix [4]. The pathophysiology of cervical incompetence is not clearly defined. It is probably not one specific abnormality rather a spectrum of different degree of anatomic deficiency including congenital connective tissue defects. Multiple small laceration as a result of trauma, gross cervical tears, and congenital malformations, such as those seen in patients with diethylstilbestrol exposure [5]. Varying patterns of uterine contractions as a result of the effects of physical activity and multiple gestation further contribute incompetence. The final common pathway for cervical incompetence as a result of these factors is painless dilatation of the cervix [6]. As in all areas of medicine, a correct diagnosis is essential to obtain the optimal therapeutic result. Therefore, pregnancy evaluation and appropriate selection of patients for operation are paramount [6].

Special attention needs to be paid to the cervix starting at 20 weeks with a vaginal ultrasound to determine cervical length, a length less than $2.0 \mathrm{~cm}$ is abnormal and will alert you to intensify management and possibly to place a cerclage in the cervix [1]. There is no apparent benefit of hospital submission for bed rest in multiple gestation if there are no complications. These also do not appear to be any benefit to placing a cervical cerclage unless there is shortening of the cervix [4]. Our data suggest that the length of cervix is an indirect indicator of its competence variable. The length of the cervix is directly correlated with the duration of pregnancy. The shorter the cervix, the greater the likelihood of the preterm delivery. Theories of premature labor based on an understanding of the cervix as uniformity competent may underestimate the importance of the cervix, and overestimate the rate of uterine activity, in the pathogenesis of prematurity [6]. Reassessment of the cervix as a structure with variable performance along a continuum supports a theory of spontaneous prematurity as a multifactorial phenomenon in which the cervix importance of decreased cervical resistance increases as the length of gestation at the time of preterm delivery decreases [6]. Obstetricians should be comfortable using the cervical length as an estimate of the risk of prematurity since many obstetrical tests are based on percentiles although funneling was predictive of prematurity. 
The ability to identify this phenomenon was not consistent among our study. Cervical length was more consistently measure than funneling and performed as well as or better than funneling in terms of sensitivity and predictive value. Transabdominal ultrasonography is unsatisfactory for measurement of the cervix because of technical drawbacks that produce inaccurate or poor-quality images. Vaginal ultrasonography produced good images in our study and was well accepted by patients. Ultimately, we expect that cervical ultrasonography will be used to evaluate women with a historical risk factor, and to select candidates for clinical trials to evaluate cerclage [6].

\section{Conclusion}

Cervical assessment during routine ultrasound in multiple pregnancy at $16-20$ weeks seems to be useful for prediction of preterm delivery and counseling the patient without risk factors regarding cervical cerclage. Because of restricted number of patients, we suggest further study with bigger sample and prospective trial for valued conclusion.

\section{Conflicts of Interest}

The authors declare no conflicts of interest regarding the publication of this paper.

\section{References}

[1] Elliott, J.P. (2003) Multiple Gestation. Copyright 2000 BestDoctors.com. All Rights Reserved.

[2] Ressel, G.W. (2004) Guidelines, Medical Screening, Reproductive System, Gynecology, Ultrasonic Imaging, Obstetrics, Women. ACOG Releases Bulletin on Managing Cervical Insufficiency. American Family Physician, Kansas City, Vol. 69, p. 436.

[3] National Institute of Health. (2001) Shortened Cervix in Second Trimester Possible Warning Sign for Premature Birth. Embargoed by Journal, 18 September.

[4] Wu, M.Y., Yang, Y.S., Huang, S.C., Lee, T.Y. and Ho, H.N. (1996) Emergent and Elective Cervical Cerclage for Cervical Incompetence. International Journal of $G y$ necology \& Obstetrics, 54, 23-29. https://doi.org/10.1016/0020-7292(96)02675-6

[5] Friedenm, F.J., Ordorica, S.A., Hoskins, I.A. and Young, B.K. (1990) The Shirodkar Operation. A Reappraisal. American Journal of Obstetrics and Gynecology, 163, 830-833. https://doi.org/10.1016/0002-9378(90)91078-Q

[6] Lams, J.D., Goldenberg, R.L., Meis, P.J., Mercer, B.M., Moawad, A., Das, A., Thorn, E., McNellis, D., Ciopper, R.L., Johnson, F., Roberts, J.M. and the National Institute of Child Health and Human Development Material Fetal Medicine Unit Network. (1996) The Length of the Cervix and the Risk of Spontaneous Premature Delivery. The New England Journal of Medicine, 334, 567-572. https://doi.org/10.1056/NEJM199602293340904 